\title{
The utility of the prehospital electrocardiogram
}

\author{
Matthew T. Davis, MD, MSc ${ }^{*}{ }^{\dagger}$; Adam Dukelow, MD, MHSc, FRCPC, $\mathrm{CHE}^{*}{ }^{\dagger}$; \\ Shelley McLeod, BScHons, MSc** Severo Rodriguez, MSc ${ }^{\dagger}$; Michael Lewell, MD, FRCPC*
}

\section{ABSTRACT}

Objectives: The 12-lead electrocardiogram (ECG) can capture valuable information in the prehospital setting. By the time patients are assessed by an emergency department (ED) physician, their symptoms and any ECG changes may have resolved. We sought to determine whether the prehospital electrocardiogram ( $p E C G$ ) could influence ED management and how often the pECG was available to and reviewed by the ED physician.

Methods: A retrospective medical record review was conducted on a random sample of patients $\geq 18$ years who had a prehospital 12-lead ECG and were transported to one of two tertiary care centres. Data were recorded onto a standardized data extraction tool. Three investigators independently compared the pECG to the first ECG obtained in the ED after patient arrival at the hospital. Any abnormalities not present on the ED ECG were adjudicated to ascertain whether they had the potential to change ED management.

Results: Of 115 ambulance runs selected, 47 had no pECG attached to the ambulance call record (ACR) and another 5 were excluded (one ST elevation myocardial infarction, one cardiac arrest, three ACR missing). Of the 63 pECGs reviewed, $16(25 \%)$ showed changes not apparent on the initial ED ECG $(\kappa=0.83 ; 95 \% \mathrm{Cl} 0.74-0.93)$, of which 12 had differences that might influence ED management $(\kappa=0.76$; $95 \% \mathrm{Cl}$ 0.72-0.82). Only one hospital record contained a copy of the $p E C G$, despite the current protocol that paramedics print two copies of the pECG on arrival in the ED (one copy for the ACR and one to be handed to the medical personnel). None of 110 ED charts documented that the pECG was reviewed by the ED physician.

Conclusion: The pECG has the potential to influence ED management. Improvement in paramedic and physician documentation and a formal pECG handover process appear necessary.

\section{RÉSUMÉ}

Objectifs : L'électrocardiogramme (ECG) à 12 dérivations peut fournir des renseignements précieux en contexte de préhospitalisation. En effet, avant que les patients n'aient été évalués par un médecin du service des urgences (SU), leurs symptômes et toute modification de l'ECG pourraient avoir été déjà résolus. Nous cherchions donc à déterminer si l'électrocardiogramme en préhospitalisation (ECGp) pouvait influencer la prise en charge du patient au SU et à quelle fréquence il était mis à la disponibilité du médecin du SU afin qu'il puisse l'étudier.

Méthodes : Un examen rétrospectif des dossiers médicaux a été mené sur un échantillon aléatoire de patients de plus de 18 ans soumis à un ECG à 12 dérivations et transportés dans un des deux centres de soins tertiaires. Les données ont été consignées sur un instrument normalisé d'extraction de données. Trois chercheurs ont comparé séparément I'ECGp avec le premier ECG obtenu au SU après I'arrivée du patient à I'hôpital. Toute anomalie absente de I'ECG du SU a été examinée afin de vérifier si elle pouvait avoir modifié la prise en charge du patient par le SU.

Résultats : Pour 115 transports par ambulance sélectionnés, il n'y avait pas d'ECGp annexé au rapport de sortie d'ambulance (RSA) dans 47 cas; 5 autres ont été écartés (un cas d'infarctus du myocarde avec élévation du segment ST, un arrêt cardiaque et trois RSA manquants). Pour les 63 ECGp restants examinés, $16(25 \%)$ ont révélé des changements non apparents sur I'ECG initial du SU (k 5 0,83; IC à 95\% : 0,74-0,93) et 12 d'entre eux présentaient des différences susceptibles d'influencer la prise en charge du patient par le SU (k 5 0,76; IC à 95\%: 0,72-0,82). Un seul dossier d'hôpital contenait une copie de l'ECGp, malgré le protocole en vigueur voulant que les ambulanciers l'impriment en deux exemplaires à leur arrivée au SU (I'un pour le RSA et l'autre à remettre au personnel médical). L'examen de I'ECGp par l'urgentiste n'était consigné dans aucun des 110 dossiers du SU.

From *Division of Emergency Medicine, Department of Medicine, The University of Western Ontario, London ON; and ${ }^{\dagger}$ Southwest Ontario Regional Base Hospital Program, London Health Sciences Corporation, London, ON.

Correspondence to: Dr. Matthew Davis, E1-100 Westminster Tower, 800 Commissioners Road East, London, ON N6A 5W9; Phone: (519) 6858500 ext. 76089; Fax: (519) 667-6769.

Submitted April 24, 2010; Revised August 15, 2010; Accepted September 19, 2010.

This article has been peer reviewed.

(C) Canadian Association of Emergency Physicians 
Conclusion : L'ECGp peut influencer la prise en charge d'un patient par le SU. L'amélioration de la documentation par les ambulanciers et les médecins et un processus officiel de transmission de l'ECGp semblent s'imposer.
Keywords: acute coronary syndrome, electrocardiogram, emergency medical services, prehospital
The development of portable 12-lead electrocardiogram (ECG) machines in the 1980s allowed the widespread practice of obtaining prehospital electrocardiograms (pECGs) by numerous emergency medical services (EMS). The American Heart Association made the implementation of a pECG program a class I recommendation in 2000. ${ }^{1}$ The 2004 and 2009 American Heart Association/American College of Cardiology guidelines for the management of patients with ST elevation myocardial infarction (STEMI) recommend that a pECG be obtained in patients suspected of having acute coronary syndrome (ACS; class IIa). ${ }^{2}$ Additionally, the National Association of EMS Physicians and others have made similar recommendations. $^{3-6}$ Based on data from 2009, 47\% of paramedics in Canada are trained to obtain a pECG. ${ }^{\text {? }}$

Studies have shown that EMS providers can acquire diagnostic quality pECGs without delaying hospital transport appreciably. ${ }^{8-12}$ Research has also demonstrated the benefits of pECGs for decreasing door-todrug time and door-to-balloon time in patients with STEMI. ${ }^{8,13-15}$ However, very few studies have examined the utility of the pECG in the emergency department (ED) management of patients not experiencing a STEMI. Valuable diagnostic information can be captured by the pECG before any prehospital interventions occur..$^{12,16}$ By the time patients are evaluated by an ED physician, their symptoms and any ECG changes may have resolved. The primary objective of this study was to identify clinically significant abnormalities present on the pECG that were not apparent on the initial ED ECG that could influence ED management. The secondary objective was to determine how often pECGs were available to and reviewed by the ED physician.

\section{METHODS}

A retrospective medical record review was conducted for a random sample of 115 patients who had a 12-lead pECG and were transported to one of two academic tertiary care EDs (combined annual volume 150,000) in London, Ontario. A list of all pECGs performed by paramedics between November 1, 2008, and October
31, 2009, was generated from the Southwest Ontario Regional Base Hospital Program Inofas Integrated Systems database, which contains information collected from the ambulance call record (ACR). A computer-based random number generator was used to select the 115 patients. Patients with an out-of hospital STEMI or in cardiac arrest on EMS arrival were excluded. EMS in London are provided by Thames EMS, a privately contracted service that has approximately 40,000 patient contacts per year in Middlesex County. The study protocol was approved by the Health Sciences Research Ethics Board at The University of Western Ontario.

Both primary care and advanced care paramedics are trained to acquire and interpret 12-lead pECGs during an Ontario Base Hospital Medical Advisory Committee 12-lead ECG training course created by the Education Subcommittee and endorsed by the provincial Medical Advisory Committee.

Paramedics acquired 12-lead pECGs using a batterypowered Zoll M Series 12-lead ECG (Zoll, Chelmsford, MA) with an industry standard GE Marquette 12SL Interpretive ECG algorithm. All Thames EMS ambulances were equipped with the Zoll devices. By protocol, paramedics can obtain 12lead ECGs in alert patients $\geq 40 \mathrm{~kg}$ who were experiencing chest pain, their usual angina, or other symptoms consistent with cardiac ischemia and in patients with a difficult to interpret rhythm on threeor five-lead ECG monitoring.

At the time of the study, there was no formal pECG handover policy at either ED. Paramedics were required to print two copies of the pECG at the time of hospital arrival: one copy for the ACR and one to be handed to the nurse during transfer of care.

Prior to any data abstraction, all study variables were defined by the research team and a standardized data collection tool was developed. Time to initial ED ECG was defined as the time interval between ED registration and the first ECG obtained at the hospital. Prehospital treatment was defined as any medication (including oxygen) that was administered by paramedics prior to patient care handover in the ED. 
The pECGs were photocopied from the patient's hospital record, including the ACR and the nursing record. The initial ED ECG was printed from the MUSE Cardiology Information System. The pECGs were independently reviewed by two Fellow of the Royal College of Physicians-certified emergency physicians and one senior emergency medicine resident who were blinded to the clinical history and outcome of the patient. pECGs were compared to the first ED ECG to determine if there were any ischemic changes (regional ST depression $\geq 1 \mathrm{~mm}$, regional T-wave inversion $\geq 3 \mathrm{~mm}$, or left bundle branch block) or clinically significant arrhythmias (those requiring treatment or investigations) not present on the initial ED ECG. If an abnormality was identified only on the pECG, reviewers then determined if it had the potential to change ED management (investigations, treatment, inpatient referral). Discrepancies were resolved by consensus. Reviewers also determined if the pECG was of poor quality (wandering baseline, missing leads, poor print quality and/or artifact).

Data were entered directly into a study-specific Microsoft Excel database (Microsoft Corporation, Redmond, WA). Reviewer interrater reliability was calculated using Cohen's kappa ( $(\kappa)$ statistics. Descriptive statistics were summarized using means and standard deviations. Statistical analysis was conducted using Stata 11.0 (StataCorp LP, College Station, TX).

\section{RESULTS}

From November 1, 2008, to October 31, 2009, 2,203 pECGs were performed by paramedics. Of the 115 randomly selected patients, 5 were excluded (one cardiac arrest, one STEMI, three ACRs missing), leaving 110 patients included in the analysis. The mean (SD) age of included patients was 67.7 (17.0) years, and $52 \%$ were female. The median (interquartile range) time to the initial ED ECG was $15(9,23)$ minutes, and 33 patients $(30 \%)$ had an ED ECG obtained within 10 minutes of ED registration (Table 1).

Figure 1 illustrates a flow diagram of all included subjects and the potential influence that the pECG would have on the management of the patient in the ED. We found that 47 of $110(43 \%)$ patients' charts did not have the pECG included on the ACR. Only 1 of 110 ED nursing records (1\%) included the pECG. None of the 110 ED physician notes explicitly documented that the pECG had been reviewed by
Table 1. Time from emergency department registration to initial electrocardiogram, by Canadian Triage and Acuity Scale

\begin{tabular}{|c|c|c|c|}
\hline CTAS & Median (min) & $\begin{array}{l}\text { Interquartile } \\
\text { range (min) }\end{array}$ & $n$ \\
\hline 1 & 10 & - & 1 \\
\hline 2 & 13 & $8-21$ & 80 \\
\hline 3 & 17 & $14-43$ & 29 \\
\hline $\begin{array}{l}\text { All CTAS } \\
\text { scores }\end{array}$ & 15 & $9-23$ & 110 \\
\hline
\end{tabular}

the physician. Seventeen (15.5\%) pECGs were deemed by the reviewers to be of poor quality $(\kappa=1)$ owing to wandering baseline $(n=7)$, artifact $(n=6)$, or faint printing/poor ink quality $(n=4)$.

Of the 63 pECGs reviewed, 16 (25\%) showed changes not present on the initial ED ECG $(\kappa=$ 0.83 ; 95\% CI $0.74-0.93)$. Of those, 12 had differences that might influence ED management $(\kappa=0.76 ; 95 \%$ CI $0.72-0.82$ ). All of the differences were ischemic changes that were not present on the initial ED ECG (Table 2). Of these 12 patients, all 12 had received

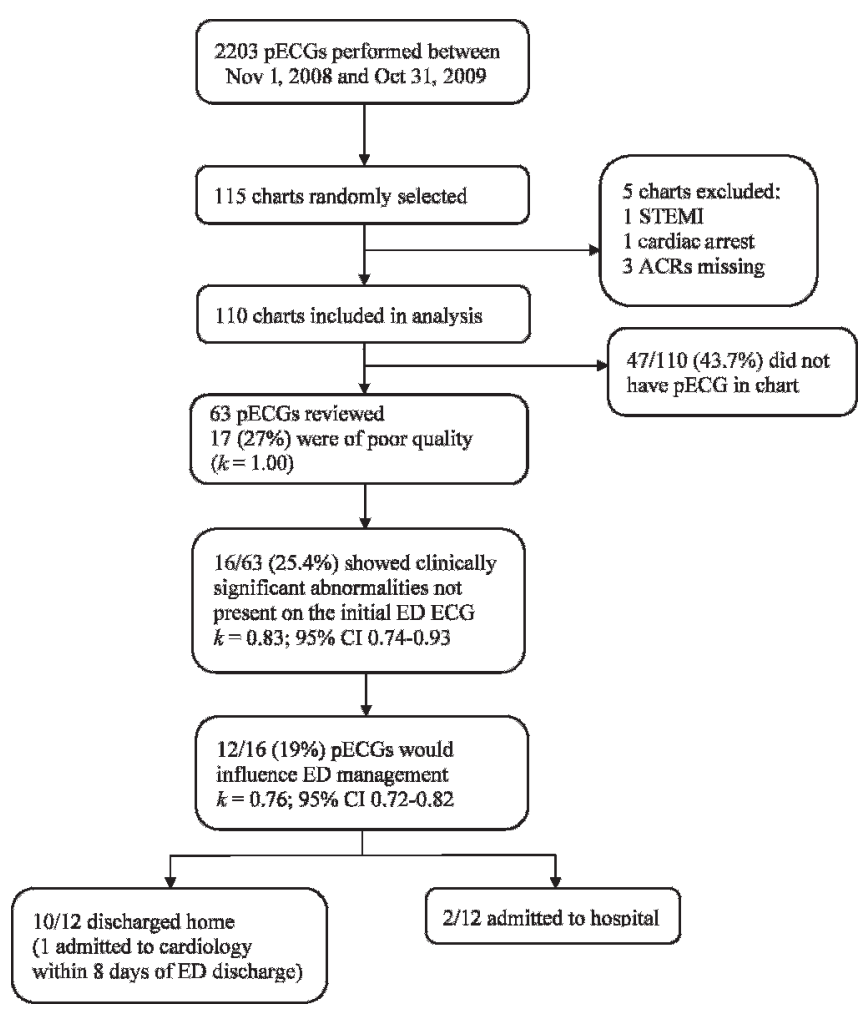

Figure 1. Flow diagram of study subjects. $A C R=$ ambulance call record; $\mathrm{ED}=$ emergency department; $\mathrm{pECG}=$ prehospital 12-lead electrocardiogram; STEMI = ST elevation myocardial infarction. 


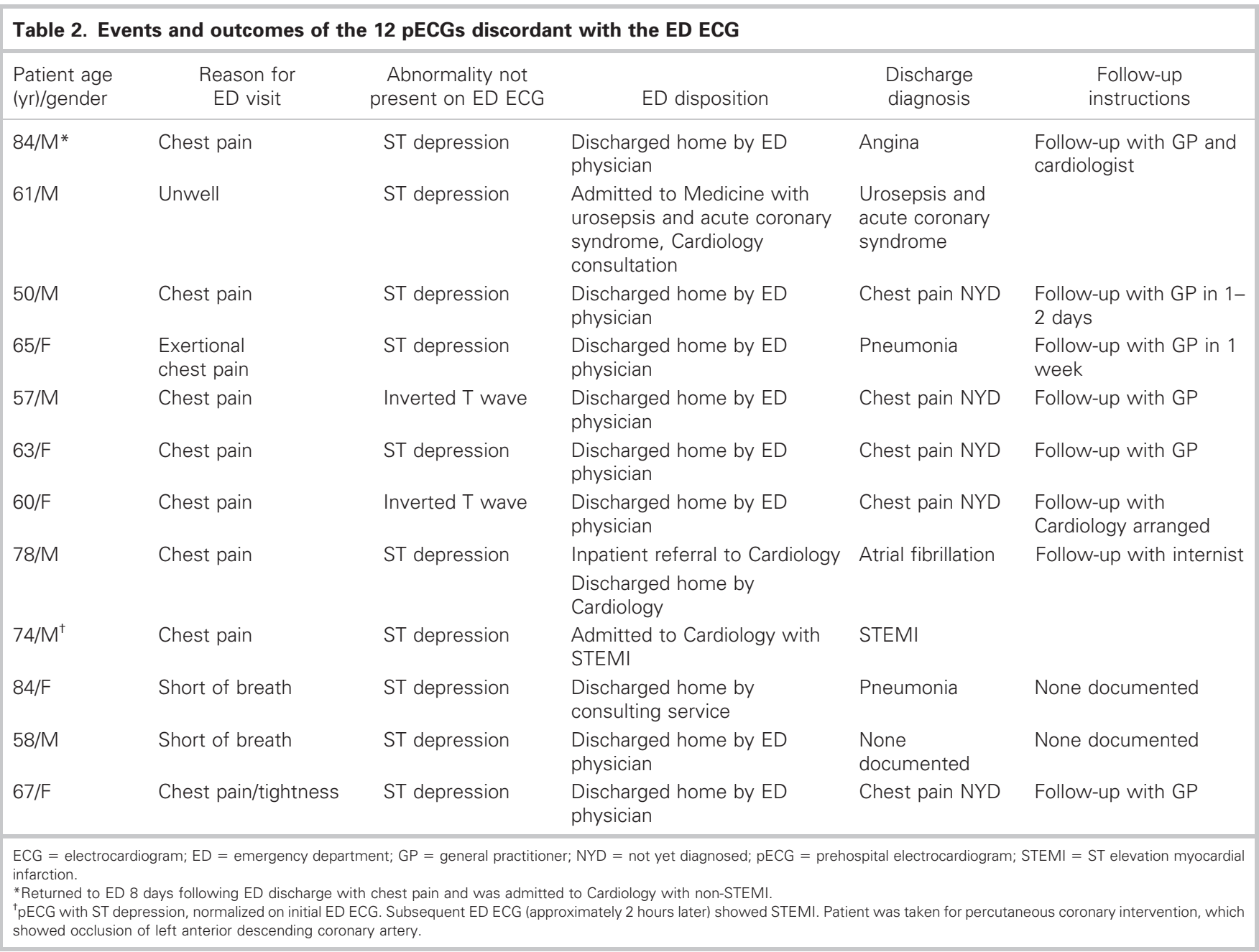

$100 \%$ oxygen in the prehospital setting, 10 acetylsalicylic acid (ASA), 4 nitroglycerin spray, and 2 salbutamol prior to arrival in the ED. Of the 12 patients, 10 were discharged home by the ED physician, 1 of whom was subsequently admitted to the cardiology service within 8 days of ED discharge.

\section{DISCUSSION}

The pECG is an important tool in the management of patients suspected of having ACS. It allows for the identification of STEMI, ischemic changes, or arrhythmias that may not be present on the ED ECG and for the triaging of chest pain patients who arrive by ambulance. ${ }^{12,16}$ We found that nearly one in four pECGs has the potential to influence the ED management of patients, mostly by identifying ischemic changes not seen on the initial ED ECG. Turnipseed and colleagues found that $17 \%$ of pECGs demonstrated
non-STEMI injury patterns (ST depression, T-wave inversion, or left branch bundle block). ${ }^{12}$ However, the authors did not address whether these changes were present on the initial ED ECG or if the pECG had any influence on the management decision of the ED physicians.

The importance of diagnosing ACS in the prehospital setting before any interventions take place is apparent. Paramedics in our county administer oxygen, ASA, and nitroglycerin spray to selected chest pain patients. These prehospital interventions may reverse the symptoms and ECG signs of ischemia prior to arrival in the ED, yet emergency physicians may place undue emphasis on the ED ECG alone. Emergency physicians discharged 10 of 12 patients who had ischemic changes seen only on their pECG. All 12 had received interventions prior to ED arrival. There was no documentation in these cases, or indeed any of the charts reviewed, indicating that the pECG had 
been reviewed by the ED physician. Cudnik and colleagues reported that having a pECG was not associated with an increase in the ED use of ASA, $\beta$ blocker, or heparin, nor was it associated with the ED length of stay. ${ }^{17}$

Our study highlights the need for a formal pECG handover system in our ED. During the study period, paramedics were required to print two copies of the pECG on arrival at the hospital. In nearly half of the ACRs reviewed, the pECG was missing. Only 1 of the 110 charts reviewed included a pECG attached to the patient's ED nursing record. Interestingly, there was not a single incidence of ED physicians documenting that the pECG was reviewed in any of the 110 reviewed charts. Omitting the review and interpretation of the pECG represents a serious information gap with degradation of clinical information at the transfer of care from prehospital to hospital personnel. To our knowledge, there are no studies that compare pECG handover systems.

Guidelines recommend that all patients experiencing chest pain have an ECG performed within 10 minutes of arrival in the ED. ${ }^{2}$ Only one-third of our patients met this threshold, comparable to the CRUSADE Initiative. ${ }^{13}$ The pECG, being available on arrival, has the potential to shorten delays in treatment when used as a diagnostic tool.

The pECGs did not capture any arrhythmias that were not present on the ED ECG. Furthermore, onequarter of the analyzed pECGs were of poor quality, which is much higher than previously reported. ${ }^{12,18}$

This study was conducted within a single EMS system and at two academic tertiary care EDs, and the results may not be generalizable. The retrospective nature of this study limited our ability to measure the importance of the pECG for patient care. Despite this limitation, the interobserver agreement was very good that the pECG in isolation had the potential to influence the ED management.

The pECG was missing from nearly half of the charts reviewed, which may have biased our sample. Had the pECG altered ED management, however, one might expect that such cases would be more likely to have retained a copy for the medical record or made a note of this influence in the medical record. We were unable to capture how many pECGs were actually reviewed by the ED physician. However, we chose the conservative assumption that if there was no documentation of the pECG interpretation in the ED chart, the pECG had not been reviewed.

\section{CONCLUSION}

The pECG is a valuable tool that can potentially influence the ED management of patients with ACS. Emergency physicians should make use of the pECG when it is available. We found the quality, documentation, and archiving of the pECG in the medical record to be poor. A formal pECG handover system appears necessary to ensure that the pECG is available to the ED physician and becomes a permanent record in the patient's hospital chart.

Competing interests: None declared.

\section{REFERENCES}

1. Guidelines 2000 for Cardiopulmonary Resuscitation and Emergency Cardiovascular Care. Part 7: the era of reperfusion: section 1: acute coronary syndromes (acute myocardial infarction). The American Heart Association in collaboration with the International Liaison Committee on Resuscitation. Circulation 2000;102(8 Suppl):I136-9.

2. Antman EM, Anbe DT, Armstrong PW, et al. ACC/AHA guidelines for the management of patients with ST elevation myocardial infarction - executive summary: a report of the American College of Cardiology/American Heart Association Task Force on Practice Guidelines (Writing Committee to Revise the 1999 Guidelines for the Management of Patients With Acute Myocardial Infarction). Circulation 2004;110:588-636, doi: 10.1161/ 01.CIR.0000134791.68010.FA.

3. Crocco TJ, Sayre MR, Aufderheide TP. Prehospital triage of chest pain patients. Prehosp Emerg Care 2002;6:224-8, doi: 10.1080/10903120290938599.

4. Jacobs AK, Antman EM, Faxon DP, et al. Development of systems of care for ST-elevation myocardial infarction patients: executive summary. Circulation 2007;116:217-30, doi: 10.1161/CIRCULATIONAHA.107.184043.

5. Dracup K, Alonzo AA, Atkins JM, et al. The physician's role in minimizing prehospital delay in patients at high risk for acute myocardial infarction: recommendations from the National Heart Attack Alert Program: Working Group on Educational Strategies to Prevent Prehospital Delay in Patients at High Risk for Acute Myocardial Infarction. Ann Intern Med 1997;126:645-51.

6. Emergency department: rapid identification and treatment of patients with acute myocardial infarction: National Heart Attack Alert Program Coordinating Committee, 60 minutes to Treatment Working Group. Ann Emerg Med 1994;23: 311-29, doi: 10.1016/S0196-0644(94)70045-1.

7. Schull MJ, Vaillancourt S, Donovan L, et al. Underuse of prehospital strategies to reduce time to reperfusion for STelevation myocardial infarction patients in 5 Canadian provinces. CFEM 2009;11:473-80. 
8. Morrison LJ, Brooks S, Sawadsky B, et al. Prehospital 12lead electrocardiogram impact on acute myocardial infarction treatment times and mortality: a systematic review. Acad Emerg Med 2006;13:84-9, doi: 10.1111/j.1553-2712.2006. tb00989.x.

9. Karagounis L, Ipsen SK, Jessop MR, et al. Impact of fieldtransmitted electrocardiography on time to in-hospital thrombolytic therapy in acute myocardial infarction. Am $\mathcal{F}$ Cardiol 1990;66:786-91, doi: 10.1016/0002-9149(90)90352-2.

10. Kudenchuk PJ, Maynard C, Cobb LA, et al. Utility of the prehospital electrocardiogram in diagnosing acute coronary syndromes: the Myocardial Infarction Triage and Intervention (MITI) Project. 7 Am Coll Cardiol 1998;32:1727, doi: 10.1016/S0735-1097(98)00175-2.

11. Kereiakes DJ, Weaver WD, Anderson JL, et al. Time delays in the diagnosis and treatment of acute myocardial infarction: a tale of eight cities. Report from the Pre-hospital Study Group and the Cincinnati Heart Project. Am Heart 7 1990;120:773-80, doi: 10.1016/0002-8703(90)90192-Z.

12. Turnipseed SD, Amsterdam EA, Laurin EG, et al. Frequency of non-ST segment elevation injury patterns on prehospital electrocardiograms. Prehosp Emerg Care 2010;14: 1-5, doi: 10.3109/10903120903144924.

13. Diercks DB, Kontos MC, Chen AY, et al. Utilization and impact of pre-hospital electrocardiograms for patients with acute ST segment elevation myocardial infarction: data from the NCDR (National Cardiovascular Data Registry) ACTION (Acute Coronary Treatment and Intervention
Outcomes Network) Registry. 7 Am Coll Cardiol 2009;53: 161-6, doi: 10.1016/j.jacc.2008.09.030.

14. Adams GL, Campbell PT, Adams JL. Effectiveness of prehospital wireless transmission of electrocardiograms to a cardiologist via hand-held device for patients with acute myocardial infarction. Am 7 Cardiol 2006;98:1160-4, doi: 10.1016/j.amjcard.2006.05.042.

15. Brainard AH, Raynovich W, Tandberg D, et al. The prehospital 12-lead electrocardiogram's effect on time of initiation of reperfusion therapy: a systematic review and meta-analysis of existing literature. Am 7 Emerg Med 2005; 23:351-6, doi: 10.1016/j.ajem.2005.02.004.

16. Drew BJ, Sommargren CE, Schindler DM, et al. Novel electrocardiogram configurations and transmission procedures in the prehospital setting: effect on ischemia and arrhythmia determination. 7 Electrocardiol 2006;39(4 Suppl): S157-60, doi: 10.1016/j.jelectrocard.2006.05.033.

17. Cudnik MT, Peacock F, Diercks DB, et al. Prehospital electrocardiograms (ECGs) do not improve the process of emergency department care in hospitals with higher usage of ECGs in non-ST-segment elevation myocardial infarction patients. Clin Cardiol 2009;32:668-74, doi: 10.1002/clc. 20673

18. Millar-Craig MW, Joy AV, Adamowicz R, et al. Reduction in treatment delay by paramedic ECG diagnosis of myocardial infarction with direct CCU admission. Heart 1997;78:456-61. 